\title{
UM MODELO DE SIMULAÇÃO PARA GESTÃO DA CAPACIDADE DOS AEROPORTOS BRASILEIROS
}

\author{
Antonio Rodolfo Araujo Marcos* \\ rodolfoamarcos@gmail.com
}

Luciano Ferreira*

ferreira@ufrnet.br

*Universidade Federal do Rio Grande do Norte - Natal, RN / Brasil

http://dx.doi.org/10.1590/1413-2311.0152014.48930

Recebido em 30/04/2014

Aprovado em 19/02/2015

Disponibilizado em 01/04/2015

Avaliado pelo sistema double blind review

Revista Eletrônica de Administração

Editor: Luís Felipe Nascimento

ISSN 1413-2311 (versão on line)

Editada pela Escola de Administração da Universidade Federal do Rio Grande do Sul.

Periodicidade: Quadrimestral

Sistema requerido: Adobe Acrobat Reader.

\section{RESUMO}

A importância do setor aéreo para o desenvolvimento de um país e o crescimento da demanda aérea brasileira implica na necessidade de uma gestão eficiente dos aeroportos, buscando o equilíbrio entre a capacidade e a demanda aérea. Diante disso, este artigo apresenta um modelo em Dinâmica de Sistemas capaz de auxiliar a gestão aeroportuária brasileira em relação ao dimensionamento dos subsistemas de um aeroporto (Terminal de Passageiros, Pistas e Pátio). O modelo utiliza dados sobre demanda aérea de passageiros, número médio de passageiros por voo, demanda hora-pico, nível de serviço do terminal de passageiros e tempo médio de estacionamento das aeronaves para analisar a situação de treze aeroportos brasileiros. Por fim, através da comparação da capacidade ideal produzida pelo modelo de simulação com a capacidade real instalada, o artigo classifica a situação de cada um dos subsistemas dos aeroportos estudados em adequada, crítica e preocupante. Entre os principais resultados obtidos pode-se destacar que: a Pista é o subsistema que apresenta melhores condições, enquanto que o terminal de passageiros é o subsistema em pior condição; os investimentos e aumento da capacidade são insuficientes para atender uma demanda de crescimento de $5 \%$ ao ano na hora-pico.

Palavras-chave: Gestão Aeroportuária; Gestão da Demanda; Dinâmica de Sistemas; Simulação.

\section{A SIMULATION MODEL FOR CAPACITY MANAGEMENT OF THE BRAZILIAN}

\section{AIRPORTS}

ABSTRACT

REAd | Porto Alegre - Edição 80 - N 1 - janeiro/abril 2015 - p. 1-26 
Um modelo de simulação para gestão da capacidade dos aeroportos brasileiros

The importance of air transportation to the development of a country and the growth of the Brazilian airline industry imply the need for an efficient management of the airports, aiming to balance the passenger demand and the capacity of the system. Thus, this paper presents a System Dynamics model in order to assist airport management to evaluate the size of the subsystems of an airport, such as Passenger Terminals, Runways, and Patios. Using data on air passenger demand, average number of passengers per flight, peak-hour demand, service level of the passenger terminal, and average time in the patio, this paper analyzes the situation of the thirteen Brazilian airports. Finally, by comparing the simulation data with the installed capacity, this paper classifies the situation of each subsystem of the airports studied into three categories: adequate, worrying, and critical. Among the main results obtained, we can highlight different factors, such as the subsystem that presents the best condition (the Runway), while the Passenger Terminal is the one in the worst condition. The investments and the capacity increases are insufficient to meet the passenger demand in the peak-hour with an annual growth rate of $5 \%$.

Keywords: Airport; Demand Management; System Dynamics; Simulation.

\section{UN MODELO DE SIMULACIÓN PARA LA GESTIÓN DE LA CAPACIDAD DE AEROPUERTOS BRASILEÑOS}

\section{RESUMEN}

La importancia de la industria aérea para el desarrollo de un país y el crecimiento de la demanda de la aerolínea brasileña implican la necesidad de una gestión eficiente de los aeropuertos, la búsqueda del equilibrio entre la capacidad y la demanda de aire. Por lo tanto, en este trabajo se presenta un modelo de dinámica de sistemas capaces de ayudar a la administración brasileña aeropuerto en relación con el diseño de los subsistemas de un aeropuerto (Terminal de Pasajeros, Esquí y Patio) . El modelo utiliza datos sobre la demanda de transporte aéreo de pasajeros, el número promedio de pasajeros por vuelo, la demanda en horas pico, nivel de servicio y de estacionamiento de tiempo promedio de pasajeros de la terminal de aviones para analizar la situación de los trece aeropuertos. Por último, mediante la comparación de la capacidad óptima producida por la simulación con el modelo de la capacidad instalada real, el papel clasifica la situación de cada uno de los subsistemas de aeropuertos de estudios apropiados, críticos y preocupantes. Entre los principales resultados se puede observar que: carril es el subsistema que presenta las mejores condiciones, mientras que el subsistema de la terminal de pasajeros se encuentra en peores condiciones; inversiones y aumento de la capacidad es insuficiente para atender el crecimiento de la demanda de $5 \%$ anual en la hora pico.

Palabras clave: Gestión de Aeropuertos; Gestión de la Demanda; Dinámica de Sistemas; Simulación.

\section{INTRODUÇÃO}

O transporte aéreo ganhou destaque no cenário nacional por apresentar REAd | Porto Alegre - Edição 80 - N 1 - janeiro/abril 2015 - p. 1-26 
características que o torna um setor estratégico, tanto para o governo como para analistas setoriais. Para Salgado, Vassalo e Oliveira (2010) as características que mais qualificam o setor aéreo como estratégico no Brasil são a inserção internacional do país, a integração e desenvolvimento regional, a posição no Mercosul e América Latina, a importância na economia, os impactos no crescimento econômico, a interação com a indústria aeronáutica nacional, a qualificação da mão-de-obra e a geração de investimentos.

Nos últimos anos, o setor aéreo brasileiro apresentou um grande crescimento, motivado por vários fatores, entre os quais o crescimento da economia, a inclusão dos passageiros das classes B e C, a sua relação custo/benefício, a estabilidade econômica, entre outros. De acordo com dados da INFRAERO (2013), a movimentação de passageiros e o fluxo de aeronaves apresentam um crescimento acumulado entre 2003 e 2012 de $169 \%$ e $70,3 \%$, respectivamente. O estudo realizado pela Mckinsey \& Company (2010) corrobora com a tendência de crescimento do setor, apontando que até 2030, serão necessários investimentos para aumentar a capacidade atual em 2,4 vezes, ou seja, aumentar a capacidade de 130 milhões para 310 milhões de passageiros por ano. No cenário atual, também aponta que os aeroportos de Guarulhos, Viracopos e Congonhas concentram mais de $80 \%$ da margem operacional entre os aeroportos da INFRAERO, auxiliando a subsidiar os 39 aeroportos deficitários administrados pela estatal.

Portanto, o assunto gestão da capacidade aeroportuária precisa ser tratado com grande prioridade, tanto pelos gestores dos aeroportos brasileiros, como pela academia. Nos últimos anos, diferentes trabalhos acadêmicos têm sido desenvolvidos sobre o tema, a Tabela 1 apresenta uma síntese dos modelos quantitativos desenvolvidos sobre gestão aeroportuária, bem como os métodos utilizados. O método DEA (do inglês, Data Envelopement Analysis) é visivelmente o mais utilizado, tanto em trabalhos realizados por pesquisadores brasileiros, como por pesquisadores estrangeiros. A Dinâmica de Sistemas vem sendo utilizada recentemente em modelos de simulação para gestão aeroportuária, especialmente em trabalhos que procuram confrontar a demanda com a capacidade instalada (ou capacidade a ser instalada), projetar o crescimento do setor e avaliar cenários futuros.

Um dos primeiros trabalhos analisados durante o desenvolvimento desta pesquisa foi de autoria de Miller e Clarke (2007), onde os autores avaliaram o valor estratégico da infraestrutura de transporte aéreo como uma alternativa para apoiar a aplicação dos investimentos necessários para expansão de capacidade. Mais tarde, Manataki e Zógrafos (2009) desenvolveram um modelo para o aeroporto internacional de Atenas com objetivo de 
Um modelo de simulação para gestão da capacidade dos aeroportos brasileiros

encontrar o equilíbrio entre a demanda e a configuração física dos terminais. Mais recentemente, Suryani, Chou e Chen (2010) utilizaram o método para prever a demanda futura de passageiros e avaliar a capacidade da pista e do terminal de passageiros do aeroportuário de Taiwan para suportar seu crescimento em longo prazo. Por ultimo, Suryani, Chou e Chen (2012) apresentaram um modelo de previsão da demanda e avaliação da capacidade do terminal de cargas.

Tabela 1 - Trabalhos relacionados

\begin{tabular}{l|l}
\hline Método & Autores \\
\hline DEA & $\begin{array}{l}\text { Gillen e Lall (1997); Martín e Román (2001; 2006); Fernandes e Pacheco (2002; 2003; } \\
\text { 2007); Sarkis e Talluri (2004); Pacheco, Fernandes e Santos (2006); Almeida, Mariano, } \\
\text { Rebelatto (2007); Barros e Dieke (2008); Curi, Gitto e Mancuso (2010); Yu (2010); } \\
\text { Pires e Fernandes (2012), Wanke (2012) }\end{array}$ \\
\hline Multicritério & $\begin{array}{l}\text { Yeh e Yuo (2003); Mello et al. (2005); Kiyildi e Karasahin (2008); Fernandes e } \\
\text { Pacheco (2010); Kuo e Liang (2011) }\end{array}$ \\
\hline Programação Linear & $\begin{array}{l}\text { Gilbo (1993) } \\
\text { Método Estatístico }\end{array}$ \\
$\begin{array}{l}\text { Hooper e Hensher (1997), Oum, Yu e Fu (2003); Correia, Wirasinghe, Barros (2008); } \\
\text { Marazzo, Scherre e Fernandes (2010); Assaf, Gillen e Barros (2012) }\end{array}$ \\
\hline Simulação & $\begin{array}{l}\text { Miller e Clarke (2007), Manataki e Zografos (2009; 2010); Suryani, Chou e Chen } \\
\text { (2010), Suryani, Chou e Chen (2012) }\end{array}$ \\
\hline
\end{tabular}

Fonte: Elaborado pelos autores

Ao analisar os trabalhos brasileiros publicados sobre o tema, tanto em periódicos do Brasil, como do exterior, verifica-se que os mesmos não consideram a avaliação e o crescimento do setor em longo prazo, são limitados a determinados recortes temporais e a dados históricos e não fazem projeções futuras. O objetivo deste trabalho é ampliar o escopo dos trabalhos prévios sobre avaliação de capacidade dos aeroportos e adaptá-los para a realidade brasileira, considerando que a capacidade aeroportuária deve ser explicada através das seguintes dimensões: Terminal de Passageiros, Pista e Pátio, como defendem Carvalho (2006), Horonjeff et al. (2010), Ashford, Mumayiz e Wright (2011), e a Agência Nacional de Aviação Civil (ANAC) por meio de suas resoluções.

Para atingir este objetivo, este artigo apresenta o projeto e a validação de um modelo em Dinâmica de Sistemas capaz de auxiliar no dimensionamento da capacidade dos aeroportos brasileiros, tanto em relação à capacidade das pistas, como em relação à capacidade do terminal de passageiros e do pátio de estacionamento das aeronaves, a partir da demanda por passagens aéreas, do número médio de passageiros por vôo, do índice de concentração da hora-pico, do nível de serviço do terminal de passageiros e do tempo médio REAd | Porto Alegre - Edição 80 - $\mathrm{N}^{\circ} 1$ - janeiro/abril 2015 - p. 1-26 
de ocupação do pátio pelas aeronaves. O modelo projetado permite avaliar a capacidade dos aeroportos brasileiros a médio e longo prazo, característica fundamental diante do tempo necessário para a implementação de qualquer adequação na infraestrutura aeroportuária, bem como da magnitude dos investimentos associados.

A realização de um estudo com as características citadas, mesmo tendo um caráter macro e não realizando análises detalhadas sobre todos os setores de um aeroporto e suas respectivas funções, é capaz de colaborar com as tomadas de decisões dos administradores aeroportuários, auxiliar na diminuição de gargalos, avaliar a capacidade instalada, avaliar o resultado dos investimentos realizados, além de servir como uma ferramenta de análise de cenários do setor em questão.

O restante deste trabalho está estruturado da seguinte forma: a seção 2 apresenta uma breve contextualização sobre o setor aeroportuário e sobre os conceitos de gestão da capacidade e demanda, envolvidos no trabalho; a seção 3 apresenta os passos metodológicos seguidos para a construção do modelo; a seção 4 apresenta a formulação do modelo e a relação entre as principais variáveis envolvidas; a seção 5 apresenta os resultados e a análise dos cenários; e, por fim, a seção 6 está reservada para as conclusões e considerações finais.

\section{CONTEXTO DO TRABALHO}

A aviação brasileira está vinculada a Secretária de Aviação Civil através dos órgãos INFRAERO e ANAC (Agência Nacional de Aviação Civil) responsáveis pela administração e regulação dos voos ocorridos no espaço aéreo brasileiro. A administração dos aeroportos é de responsabilidade da INFRAERO, constituída nos termos da Lei ${ }^{\circ}$ 5.862, de 12 de dezembro de 1972, para implantar, administrar, operar e explorar industrial e comercialmente a infraestrutura aeroportuária e de apoio à navegação aérea, além de prestar consultoria e assessoramento nas áreas de atuação e na construção de aeroportos. Atualmente, a INFRAERO administra 66 aeroportos, 69 Grupamentos de Navegação Aérea, 50 Unidades Técnicas de Aeronavegação e 34 terminais de logística de carga. As atribuições da ANAC (Agência Nacional de Aviação Civil) são regular e fiscalizar as atividades de aviação civil e de infraestrutura aeronáutica e aeroportuária. Assim, a atividade regulatória se divide em regulação técnica (segurança dos passageiros e usuários da aviação civil) e regulação econômica (monitoramento e possíveis intervenções no mercado em busca da máxima eficiência).

REAd | Porto Alegre - Edição 80 - $\mathrm{N}^{\circ} 1$ - janeiro/abril 2015 - p. 1-26 
Um modelo de simulação para gestão da capacidade dos aeroportos brasileiros

Os órgãos citados, INFRAERO e ANAC, concentram a maioria dos dados sobre a aviação civil no Brasil, formando uma base de dados apropriada para construir um panorama do setor aéreo brasileiro no que diz respeito à quantidade de passageiros, aeronaves, cargas e resultados financeiros das principais unidades aeroportuárias do país, além de apresentar informações sobre a gestão estratégica.

Os aeroportos brasileiros apresentaram índices positivos de crescimento no período entre 2003 e 2011 na movimentação de passageiros (embarque e desembarque) e fluxo de aeronaves (pousos e decolagens) nas suas unidades. A movimentação de passageiros e o fluxo de aeronaves apresentam um crescimento acumulado no período citado de, respectivamente, $152,68 \%$ e 63,87\%. Quanto à movimentação de cargas, apesar das oscilações negativas apresentadas em alguns anos, houve um aumento de $28,75 \%$ na quantidade de toneladas transportadas entre 2003 e 2011.

Dentre os 66 aeroportos administrados pela INFRAERO, somente os aeroportos localizados nos Estados das 12 cidades-sedes da Copa do Mundo de Futebol de 2014 estão incluídos no plano de investimentos do Governo Federal para obter expansão da sua capacidade, oportunizando um acréscimo significativo na movimentação de passageiros entre os estados participantes. Os investimentos previstos nesses aeroportos são descritos pelo Tribunal de Contas da União - TCU (2012), conforme Tabela 2.

Tabela 2 - Investimentos previstos nos aeroportos brasileiros

\begin{tabular}{|c|c|}
\hline Aeroporto & $\begin{array}{l}\text { Investimento Previsto } \\
\text { (em milhões de reais) }\end{array}$ \\
\hline Brasília - DF & $\mathrm{R} \$ 864 ., 70$ \\
\hline Campinas - SP & $\mathrm{R} \$ 876,90$ \\
\hline Confins - MG & $\mathrm{R} \$ 508,70$ \\
\hline Curitiba - PR & $\mathrm{R} \$ 84,50$ \\
\hline Cuiabá - MT & $\mathrm{R} \$ 91,30$ \\
\hline Fortaleza-CE & $\mathrm{R} \$ 349,80$ \\
\hline Galeão - RJ & $\mathrm{R} \$ 813,30$ \\
\hline Guarulhos - SP & $\mathrm{R} \$ 1331,70$ \\
\hline Manaus - AM & $\mathrm{R} \$ 394,10$ \\
\hline Natal - RN & $\mathrm{R} \$ 582,40$ \\
\hline Porto Alegre - RS & $\mathrm{R} \$ 579,20$ \\
\hline Recife - PE & $\mathrm{R} \$ 18,50$ \\
\hline Salvador - BA & $\mathrm{R} \$ 47,60$ \\
\hline Total & R\$ 6542,70 \\
\hline
\end{tabular}

Entre os aeroportos citados com investimentos previstos pelo TCU, o investimento para o aeroporto localizado na cidade de Natal-RN se refere à construção de um novo 
Antonio Rodolfo Araujo Marcos \& Luciano Ferreira

aeroporto. Porém, a construção é de responsabilidade de um Consórcio de empresas que irá administrar o aeroporto conforme estabelecido no leilão de concessão desse aeroporto.

Considerando a movimentação de passageiros e as unidades aeroportuárias no Brasil com previsão de investimentos segundo o TCU (2012), os 13 aeroportos citados anteriormente correspondem a aproximadamente $67,3 \%$ do tráfego de passageiros aéreos no Brasil, conforme apresentado na Tabela 3.

Tabela 3 - Movimentação de passageiros nos principais aeroportos

\begin{tabular}{l|r}
\hline \multicolumn{1}{c|}{ Aeroporto } & \multicolumn{1}{c}{ Passageiros } \\
\hline SBBR - Aeroporto Internacional de Brasília & 15.665 .045 \\
\hline SBCF - Aeroporto Internacional de Confins & 10.200 .348 \\
\hline SBCT - Aeroporto Internacional de Curitiba & 6.825 .666 \\
\hline SBCY - Aeroporto Internacional de Cuiabá & 2.761 .588 \\
\hline SBEG - Aeroporto Internacional de Manaus & 3.126 .179 \\
\hline SBFZ - Aeroporto Internacional de Fortaleza & 5.964 .223 \\
\hline SBGL - Aeroporto Internacional do Galeão & 17.491 .744 \\
\hline SBGR - Aeroporto Internacional de Guarulhos & 32.177 .594 \\
\hline SBKP - Aeroporto Internacional de Campinas & 15.665 .045 \\
\hline SBNT - Aeroporto Internacional de Natal & 2.660 .864 \\
\hline SBPA - Aeroporto Internacional de Porto Alegre & 8.261 .355 \\
\hline SBRF - Aeroporto Internacional de Recife & 6.403 .416 \\
\hline SBSV - Aeroporto Internacional de Salvador & 8.528 .837 \\
\hline Total & 191.617 .646 \\
\hline
\end{tabular}

Fonte: INFRAERO (2013)

\subsection{Gestão da Capacidade e Demanda}

O termo capacidade, em linhas gerais, refere-se a um valor quantitativo que indica o fornecimento de um serviço por um determinado período de tempo. A capacidade de um aeroporto pode ser estudada com o propósito de dimensionar a relação entre a demanda e a capacidade; para levantar necessidades de investimentos em instalações aeroportuárias; para medir objetivamente a capacidade operacional dos vários componentes do sistema aeroportuário para o tratamento de passageiros projetado e fluxos de aeronaves; e, para estimar os atrasos e outras manifestações de restrições de capacidade vividas no sistema em diferentes níveis de demanda (ASHFORD, MUMAYIZ e WRIGHT, 2011).

A capacidade de um aeroporto está relacionada com a capacidade de seus respectivos subsistemas, ou seja, depende da capacidade do Terminal de Passageiros, da Pista e do Pátio 
Um modelo de simulação para gestão da capacidade dos aeroportos brasileiros

de estacionamento das aeronaves, conforme apontam Horonjeff et al. (2010), Ashford, Mumayiz e Wright (2011), Carvalho (2006) e os órgãos brasileiros envolvidos com o transporte aéreo - INFRAERO e ANAC.

A utilização de cada subsistema do aeroporto depende da existência da demanda de passageiros, uma vez que a principal função do aeroporto está relacionada com o transporte dos mesmos. Assim, o pleno funcionamento do aeroporto com adequados níveis de serviço requer o total atendimento da demanda de passageiros.

A demanda de passageiros na hora-pico é utilizada nos modelos quantitativos para gestão aeroportuária por representar o período de tempo com maior utilização dos serviços aéreos, sendo essencial para avaliar o nível adequado de atendimento dos serviços, pois possibilita estudar o sistema sob condições limite, onde diversos problemas podem ocorrer, tais como congestionamentos, filas, atrasos, entre outras situações (WANG e PITFIELD, 1999; ANAC, 2007).

Portanto, a previsão do movimento de passageiros na hora-pico é fundamental no processo de dimensionamento dos subsistemas de um aeroporto. A literatura especializada apresenta diferentes métodos para calcular a demanda hora-pico a partir da demanda anual de passageiros, entre os quais Wang e Pitfield (1999), FAA (2013) e ANAC (2007).

Nesse sentido, Wang e Pitfield (1999) realizaram um estudo com 48 aeroportos brasileiros administrados pela INFRAERO para determinar a demanda hora-pico de projeto dos aeroportos. A partir deste trabalho, o autor define, matematicamente, a demanda horapico dos aeroportos brasileiros como uma regressão linear, com melhores resultados para os aeroportos com movimento anual superior a 200.000 passageiros, e como uma regressão não linear, com melhores resultados para os aeroportos menores.

A Federal Aviation Administration (FAA), conforme descrito por Ashford et al. (1984), utiliza o Typical Peak Hour Passenger (TPHP) para estimar o movimento de passageiros na hora-pico, onde o índice de concentração de passageiros neste horário é obtido a partir da demanda anual de passageiros. ANAC (2007) considerou o método utilizado pela FAA inadequado para o caso brasileiro e redimensionou o índice de concentração de cada uma das faixas. O método utilizado em ANAC (2007) prevê um intervalo de valores para cada faixa para contemplar as incertezas inerentes ao processo de modelagem e estimação, conforme Tabela 4. 
Antonio Rodolfo Araujo Marcos \& Luciano Ferreira

Tabela 4 - Índice da FAA para hora-pico

\begin{tabular}{l|c|c|c}
\hline Passageiros anuais & TPHP (\%) & Limite inferior (\%) & Limite superior (\%) \\
\hline Acima de 30 milhões & 0,035 & 0,024 & 0,026 \\
\hline De 20 milhões a 29,9 milhões & 0,040 & 0,026 & 0,026 \\
\hline De 10 milhões a 19,9 milhões & 0,045 & 0,026 & 0,027 \\
\hline De 1 milhão a 9,9 milhões & 0,050 & 0,027 & 0,051 \\
\hline De 500 mil a 999,9 mil & 0,080 & 0,064 & 0,068 \\
\hline De 100 mil a 499,9 mil & 0,130 & 0,068 & 0,118 \\
\hline Abaixo de 100 mil & 0,200 & 0,169 & 0,399 \\
\hline
\end{tabular}

Fonte: ANAC (2007)

\section{PROCEDIMENTOS METODOLÓGICOS}

Os modelos de simulação são representações da realidade e podem ser utilizados com bastante sucesso para apoiar a tomada de decisão. Na construção destes modelos, parte-se do princípio que é possível abstrair e reduzir a complexidade da situação problemática, representá-la formalmente em um programa de computador e, por fim, analisá-la através de diferentes experimentos.

A Dinâmica de Sistemas, método de pesquisa utilizado neste trabalho, publicada no livro Industrial Dynamics de autoria do engenheiro eletricista Jay Forrester em 1961, pode ser utilizada para estudar o comportamento de sistemas industriais para demonstrar como diretrizes, decisões, estruturas e retardos se inter-relacionam para influenciar o crescimento e a estabilidade (STERMAN, 2000). Além disso, permite representar o mundo real aceitando a complexidade, a não linearidade e estruturas de loop de feedback que são inerentes a sistemas sociais e físicos.

$\mathrm{Na}$ etapa inicial de modelagem, o Diagrama Causal, também conhecido como Diagrama de Enlace Causal, é o elemento essencial na construção de qualquer modelo em Dinâmica de Sistemas. Este tipo de diagrama consiste em um conjunto de variáveis conectadas por setas denotando influências causais entre as variáveis, ou seja, é uma representação qualitativa das relações de causa e efeito entre as variáveis envolvidas no problema de decisão.

Após a construção e validação do Diagrama Causal, um Diagrama de Estoque e Fluxo precisa ser construído para que o modelo possa ser simulado em alguma ferramenta 
Um modelo de simulação para gestão da capacidade dos aeroportos brasileiros

computacional, tal como iThink® e Vensim®, por exemplo. Este modelo é composto por estoques, fluxos, auxiliares e conectores, conforme descrição apresentada em Sterman (2000).

As seções seguintes apresentam o detalhamento das etapas metodológicas conduzidas para a realização deste trabalho, conforme sequencia listada a seguir:

1. Levantamento dos dados e análise da situação: conforme seção 3.1;

2. Construção do Diagrama Causal: conforme seção 3.2;

3. Construção do Diagrama de Estoque e Fluxo: conforme seção 3.3;

4. Testes e validação do modelo: conforme seção 3.4;

5. Avaliação de cenários: conforme seção 4.

\section{DESCRIÇÃO DO MODELO PROPOSTO}

Esta seção apresenta em detalhes a formalização do modelo de simulação proposto neste trabalho para avaliação da capacidade dos aeroportos brasileiros, conforme etapas metodológicas descritas em Sterman (2000).

\subsection{Levantamento dos dados}

Inicialmente, foram realizadas entrevistas com especialistas do setor e uma extensiva pesquisa bibliográfica sobre os principais trabalhos publicados na área de modelo quantitativos para gestão aeroportuária, cujo objetivo foi entender o funcionamento do setor e identificar possíveis lacunas existentes, conforme sistematização previamente apresentada na Tabela 1. Após, foram analisados e sistematizados dados da INFRAERO (2013), ANAC (2007) e consultorias realizadas no setor (ver, por exemplo, Mckinsey \& Company (2010)). Com isso, foi possível identificar o principal alvo desta pesquisa: a avaliação da capacidade dos aeroportos brasileiros em médio e longo prazo frente à crescente demanda do setor. Nesta etapa também foram identificadas as variáveis-chaves para o modelo de simulação e o horizonte de tempo da análise.

As principais variáveis-chaves elencadas durante o estudo e elaboração do modelo de simulação foram as seguintes: demanda anual de passageiros, capacidade do terminal de passageiros, capacidade das pistas e capacidade do pátio de um aeroporto. Já o horizonte de tempo estabelecido para validar o modelo de simulação, compreendeu os anos de 2003 a 2012, pela disponibilidade de dados para confrontar com os resultados gerados pelo modelo. 
Para os a prospecção de cenários e análises futuras, considerou-se o ano de 2020 como o marco final, em função da finalização das obras previstas para o setor e também pelas previsões de crescimento da demanda.

Nesta etapa também foi definido o escopo do conceito de capacidade aeroportuária, definida neste trabalho como sendo a capacidade de cada um dos subsistemas que compõe um aeroporto, ou seja, capacidade do terminal de passageiros, capacidade da pista e capacidade do pátio de estacionamento das aeronaves, conforme trabalhos prévios analisados, tais como Carvalho (2006), Horonjeff et al. (2010), Ashford, Mumayiz e Wright (2011), INFRAERO (2013) e ANAC (2007).

Por último, foram escolhidos os aeroportos de Brasília, Campinas, Confins, Cuiabá, Curitiba, Fortaleza, Rio de Janeiro (Galeão), São Paulo (Guarulhos), Manaus, Natal, Porto Alegre, Recife e Salvador para compor a amostra de estudo deste trabalho, pois os mesmos concentram aproximadamente $70 \%$ do tráfego de passageiros aéreos do Brasil, estão localizados em cidades-sedes da Copa do Mundo de 2014 e foram contemplados no plano de investimentos do Governo Federal para expansão de capacidade aeroportuária.

\subsection{Diagrama Causal}

O Diagrama de Enlace Causal representa as principais relações e laços reforçadores do sistema analisado. A demanda de passageiros é o elemento central do Diagrama Causal projetado neste trabalho, dado que o objetivo do atual modelo é atender completamente a demanda de passageiros em níveis adequados de serviço.

A Figura 1 apresenta o Diagrama Causal desenvolvido, o qual está dividido em três submodelos: subsistema Terminal de Passageiros, subsistema Pista e subsistema Pátio. As setas no Diagrama Causal representam uma polaridade (positiva ou negativa) para indicar o sentido da mudança na variável dependente. No modelo apresentado na Figura 1 há dois loopings em cada submodelo para manter o sistema em equilíbrio, seu objetivo é balancear a capacidade de cada subsistema em função da demanda, conforme descrito a seguir:

Excesso de Capacidade do Subsistema $\stackrel{-}{\rightarrow}$ Capacidade do Subsistema $\stackrel{+}{\rightarrow}$ Excesso de Capacidade do Subsistema Capacidade do Subsistema $\stackrel{-}{\rightarrow}$ Utilização $\stackrel{+}{\rightarrow}$ Capacidade do Subsistema 
Um modelo de simulação para gestão da capacidade dos aeroportos brasileiros

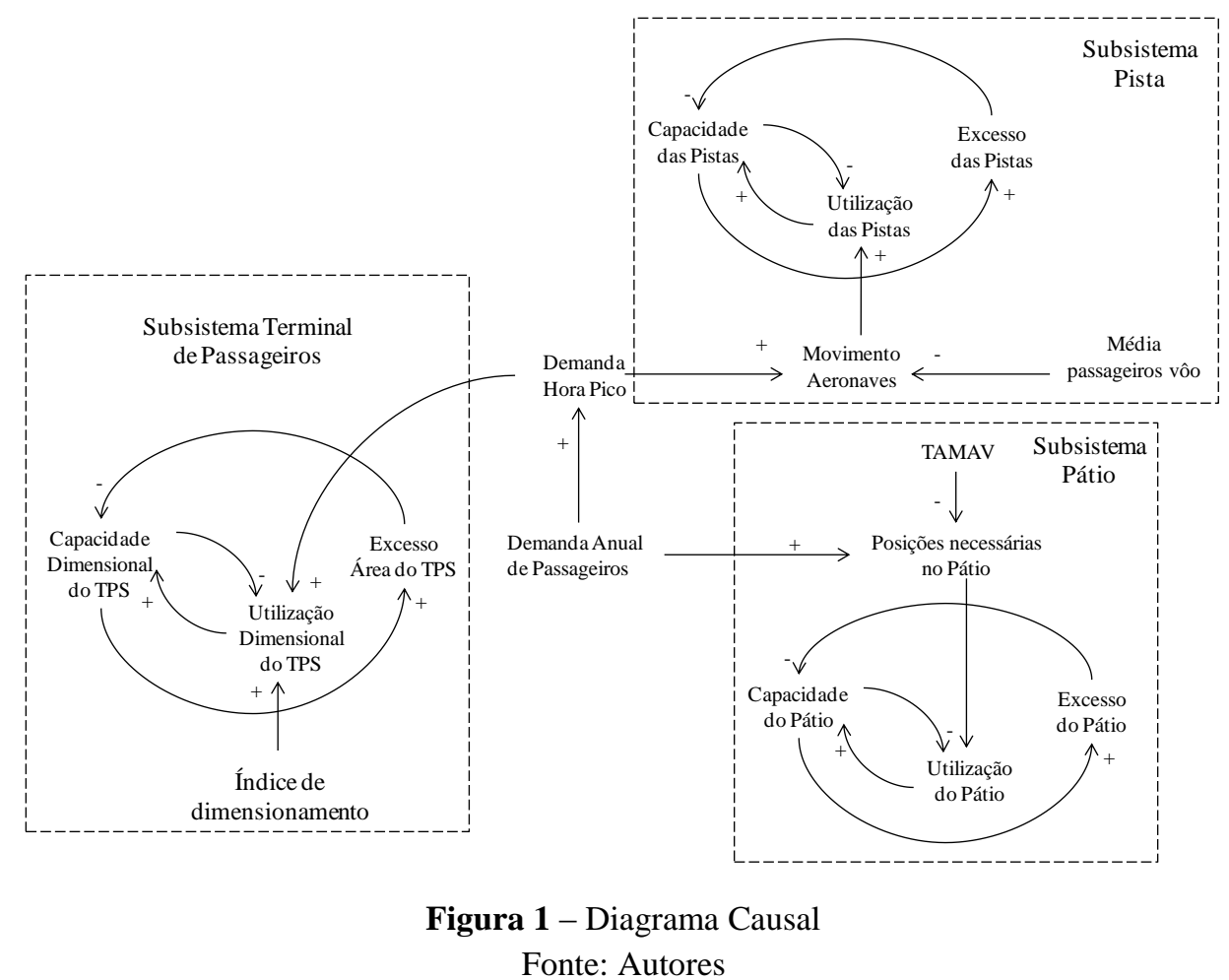

Na primeira situação, o excesso de capacidade do subsistema influencia negativamente a capacidade do sistema, ou seja, quando há excesso de capacidade (sistema sub-utilizado) o modelo busca diminuir a capacidade do subsistema e quando não há excesso de capacidade (sistema superutilizado), o modelo busca aumentar a capacidade do subsistema; por outro lado, a capacidade do subsistema influencia positivamente o excesso, ou seja, sempre que a capacidade do sistema for ultrapassada (utilização maior que 100\%), o excesso de capacidade deve ser aumentado, caso contrario, o excesso de capacidade deve ser diminuído. Na segunda situação, a capacidade do subsistema influencia negativamente sua utilização, ou seja, sempre que aumentar a capacidade, a utilização tende a diminuir; por outro lado, a utilização influencia positivamente a capacidade do subsistema, ou seja, quando aumenta a utilização, o modelo procura o equilíbrio e tende a aumentar a capacidade do subsistema.

\subsection{Diagrama de Estoque e Fluxo}

A construção do Diagrama de Estoque e Fluxo foi realizado através do software iThink $^{\circledR}$, utilizando como base o Diagrama Causal apresentada na seção anterior. O modelo desenvolvido segue os pressupostos metodológicos dos trabalhos de Carvalho (2006), Medeiros (2004), Miller e Clarke (2007) e Suryani, Chou e Chen (2010). 
Inicialmente, o modelo prevê, em cada sub-modelo, uma estrutura lógica para ajustar a capacidade dos subsistemas. Esta estrutura representa a variável Capacidade Atual como um estoque, cujo valor inicial é pré-definido pelo usuário, a partir de 1, além de um fluxo de entrada (Aumentar Capacidade) e um fluxo de saída (Diminuir Capacidade) que são responsáveis por ajustar a capacidade, conforme o valor da variável Utilização. As Equações 1 e 2 formalizam este procedimento.

Aumentar Capacidade = IF Utilização > 1 THEN $($ Capacidade Atual * Utilização)/n ELSE 0

Diminuir Capacidade = IF Utilização $<=1$ THEN (Capacidade Atual * (1-Utilização))/n ELSE 0

De acordo com a Equação 1, sempre que a utilização foi maior do que 1, a capacidade deve ser aumentada, então o fluxo de entrada Aumentar Capacidade é atualizado com o valor da multiplicação da capacidade atual pela utilização, caso contrário, quando a utilização for menor do 1, o fluxo de entrada é atualizado com o valor zero e a capacidade permanecerá com o mesmo valor.

O denominador destas equações (n) é utilizado para suavizar a convergência da capacidade para seu valor ótimo e também para evitar mudanças abruptas no seu valor, este procedimento de suavização é descrito em detalhes em Sterman (2000). Após a atualização dos valores dos fluxos de entrada e saída, são atualizados os valores da capacidade atual e da utilização do sub-modelo em análise, conforme Equações 3 e 4.

Capacidade Atual $=$ Aumentar Capacidade - Diminuir Capacidade + Capacidade Atual

Utilização = Demanda $/$ Capacidade Atual

O Diagrama de Estoque e Fluxo completo é apresentado na Figura 2, cada um dos submodelos são descritos nas seções seguintes.

\subsubsection{Submodelo Terminal de Passageiros}

O Terminal de Passageiros é responsável por interligar o lado terrestre com o lado aéreo do aeroporto. Ainda, o Terminal de Passageiros (TPS) é a edificação onde os passageiros realizam todo o processo de embarque e desembarque (ASHFORD, MUMAYIZ e WRIGHT, 2011).

Com o objetivo de proporcionar a circulação e o processamento dos serviços para os passageiros, Medeiros (2004) aponta o saguão de embarque, a sala de pré-embarque, a área para vendas e reservas de bilhetes, o check-in, a área para triagem e despacho de bagagens, a área de vistoria de segurança, a área de vistoria de passaportes, o saguão de desembarque, a área de restituição de bagagens, e a área de desembarque internacional (imigração, área de restituição de bagagens e alfândega) como os componentes do Terminal de Passageiros.

REAd | Porto Alegre - Edição 80 - N 1 - janeiro/abril 2015 - p. 1-26 
Um modelo de simulação para gestão da capacidade dos aeroportos brasileiros

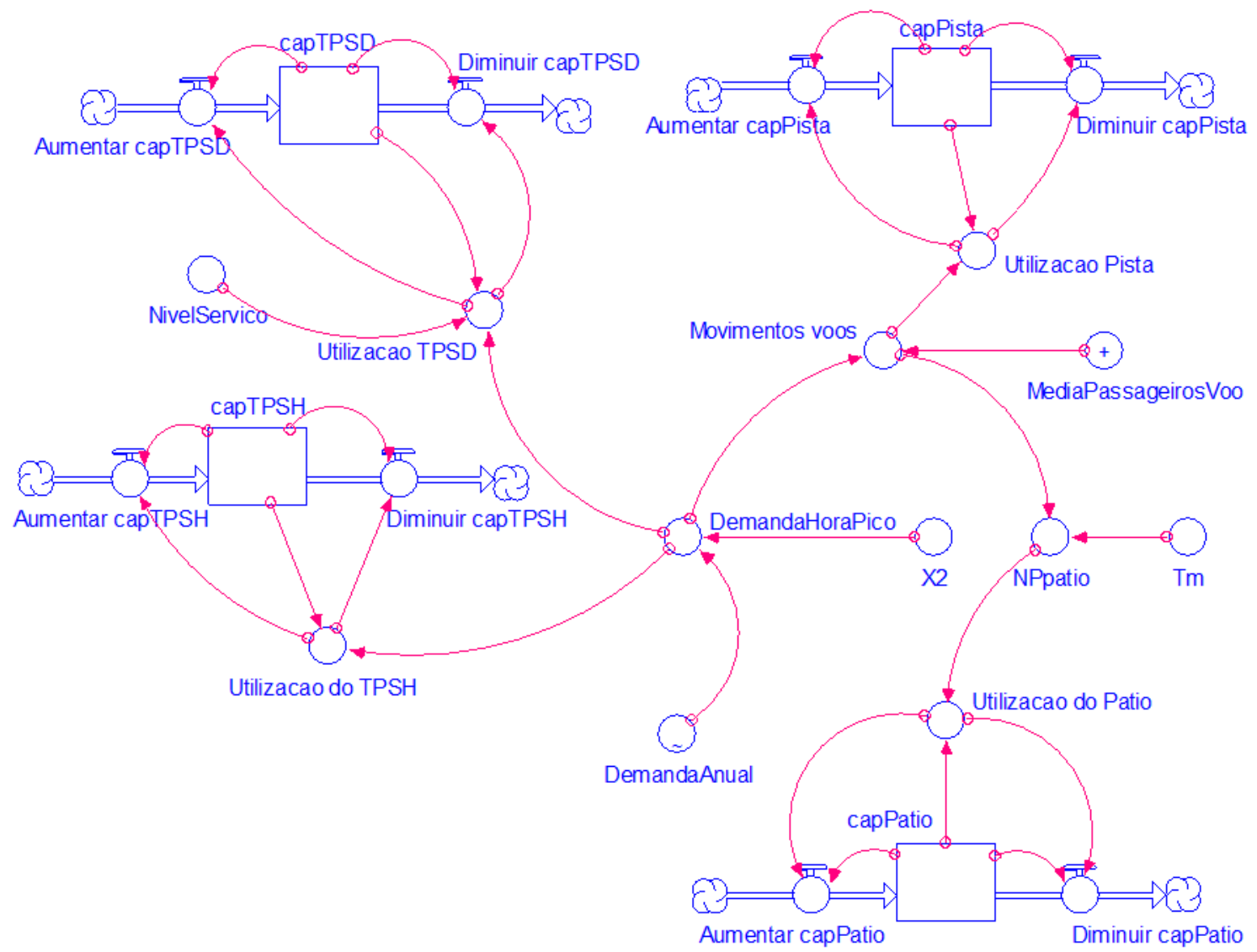

Figura 2 - Modelo proposto em Dinâmica de Sistemas Fonte: Elaborado no iThink pelos autores

O método utilizado neste trabalho para estimar a capacidade do terminal de passageiros está definido em Medeiros (2004), onde há a definição de um índice global para dimensionar o terminal de passageiros, em metros quadrados. Este índice considera a classe na qual o aeroporto pertence (internacional, doméstico ou regional) e o nível de serviço estabelecido para atender os passageiros (alto, bom ou regular), conforme Tabela 5.

Tabela 5 - Área Total do TPS

\begin{tabular}{l|c|c|c}
\hline \multirow{2}{*}{ Nível de Serviço } & \multicolumn{3}{|c}{ Tipo de aeroporto } \\
\cline { 2 - 4 } & Internacional $\left(\mathrm{m}^{2}\right)$ & Doméstico $\left(\mathrm{m}^{2}\right)$ & Regional $\left(\mathrm{m}^{2}\right)$ \\
\hline Alto & 25,00 & 18,00 & 15,00 \\
\hline Bom & 22,00 & 15,00 & 12,00 \\
\hline Regular & 18,00 & 12,00 & 10,00 \\
\hline
\end{tabular}

Fonte: Medeiros (2004)

A partir destas variáveis, a capacidade do TPS de passageiros, em metros quadros, pode ser estimada através da seguinte formalização: 


$$
\text { Capacidade Atual TPS Dimensional }=\frac{\text { Demanda Hora Pico } * \text { Indice de dimensionamento }}{\text { Utilização do TPS Dimensional }}
$$

onde, o índice de dimensionamento é escolhido pela Tabela 5, a demanda hora pico é calculada conforme descrito na seção 3.4, enquanto que a utilização é obtida pela Equação 4.

\subsubsection{Subsistema Pistas}

O subsistema Pistas envolve exclusivamente o lado aéreo do aeroporto. A capacidade da pista é definida pela quantidade de movimentos (pousos e decolagens de aeronaves) por hora, conforme Gilbo (1993) e Reynolds-Feighan e Button (1999).

A INFRAERO considera o método criado pelo órgão norte-americano Federal Aviation Administration (FAA) para calcular a capacidade do sistema de pistas dos aeroportos brasileiros, onde a capacidade das pistas é dada em função da quantidade máxima de movimentos em uma hora. Dessa forma, o cálculo da capacidade da pista pode ser formulado como segue:

Capacidade Atual Pistas $=\frac{\text { Movimentos na hora pico }}{\text { Utilização Pistas }}$

Onde

Movimentos na hora pico $=\frac{\text { Demanda Hora Pico }}{\text { Passageiros por Voo }}$

A média de vôos na hora-pico foi determinada conforme parâmetros dados em ANAC (2007) e um índice de concentração de passageiros na hora-pico foi estimada para cada aeroporto analisado, conforme parâmetro $X 2$, descrito na seção 3.4; a quantidade de passageiros por vôo deve ser estimada para cada aeroporto, levando em consideração o número de passageiros e o movimento de aeronaves na hora-pico; a utilização das pistas é calculada pela Equação 4.

\subsubsection{Subsistema Pátio}

O Pátio de um aeroporto é responsável pela ligação entre o lado terrestre e lado aéreo do aeroporto e onde ocorre a transferência de passageiros e cargas entre esses lados do aeroporto. No pátio, onde ficam estacionadas as aeronaves, a capacidade é definida pela quantidade de posições de estacionamento de aeronaves, conforme defendem Ashford, Mumayiz e Wright (2011). 
Um modelo de simulação para gestão da capacidade dos aeroportos brasileiros

O número de posições no pátio de estacionamento é diferente do número de movimentos na pista (ASHFORD et al., 1984). A capacidade da pista resultante da Equação 7 corresponde ao somatório do número de pousos e decolagens na hora-pico. Dessa forma, uma dada aeronave pousa e decola, contabilizando dois movimentos para o aeroporto, mas requer apenas uma operação no pátio. O projeto de um pátio de estacionamento deve considerar, portanto, o maior número dentre pousos e decolagens (HORONJEFF et al., 2010). Dessa forma, pode-se formalizar o número de posições de estacionamento de um aeroporto como:

Capacidade Atual Patio $=\frac{\text { Posições Necessárias do Pátio }}{\text { Utilização do Pátio }}$

onde,

Posições Necessárias do Pátio $=\frac{V * T_{m}}{60 * U}$

onde,

$V$ : movimento máximo de aeronaves na hora-pico;

$U$ : utilização do pátio de estacionamento;

$T_{m}$ : tempo médio, em minutos, de ocupação do pátio de estacionamento pelas aeronaves.

\subsection{Validação do modelo}

Após a apresentação da formulação do modelo, esta seção descreve os passos realizados para validar o modelo proposto e também para calibrar os parâmetros de entrada utilizados nas simulações, a saber: demanda anual de passageiros, número médio de passageiros por vôo, índice de concentração de passageiros na hora-pico, nível de serviço do terminal de passageiros e tempo médio de ocupação do pátio de estacionamento pelas aeronaves.

Inicialmente, o índice de concentração de passageiros na hora-pico (X2) foi validado em relação aos aeroportos estudados. Os resultados produzidos pelo modelo foram então comparados com dados reais apresentados no relatório produzido pela ANAC (2007), conforme Tabela 6. O objetivo desta etapa foi aproximar a demanda hora-pico simulada da média prevista por ANAC (2007) e, em alguns casos, não ultrapassar o valor previsto no cenário otimista.

Em seguida, o número médio passageiros por vôo foi estimado a partir dos dados que constam no relatório da ANAC (2007) levando em consideração a relação entre o número de passageiros na hora-pico e o número de movimentos de aeronaves no mesmo período. 
Antonio Rodolfo Araujo Marcos \& Luciano Ferreira

Finalmente, o nível de serviço do terminal de passageiros foi definido como "Bom" para todos os casos analisados, conforme outros trabalhos consultados, tais como Carvalho (2006) e Medeiros (2004), e o tempo médio de ocupação do pátio de estacionamento pelas aeronaves foi definido como uma distribuição normal com média de 45 minutos e desvio-padrão de 15 minutos, a partir dos coletados no site da INFRAERO (2013).

Tabela 6 - Número de passageiros na hora-pico

\begin{tabular}{l|c|c|c|c|c}
\hline \multirow{2}{*}{$\begin{array}{c}\text { Aeroporto } x \\
\text { Hora-Pico }\end{array}$} & \multicolumn{3}{|c|}{ Previsão ANAC (2007) } & \multirow{2}{*}{ X2 $(\%)$} & \multirow{2}{*}{ Simulada } \\
\cline { 2 - 4 } Brasília & Pessimista & Media & Otimista & & \\
\hline Campinas & 4041 & 4775 & 5640 & 0,03 & 4685 \\
\hline Confins & 1466 & 1739 & 2012 & 0,025 & 1785 \\
\hline Cuiabá & 617 & 753 & 919 & 0,030 & 816 \\
\hline Curitiba & 1261 & 1494 & 1768 & 0,030 & 1577 \\
\hline Fortaleza & 1228 & 1505 & 1825 & 0,025 & 1593 \\
\hline Galeão & 2784 & 3396 & 4007 & 0,020 & 3397 \\
\hline Guarulhos & 5584 & 6402 & 7219 & 0,017 & 6349 \\
\hline Manaus & 1169 & 1406 & 1679 & 0,050 & 1552 \\
\hline Natal & 815 & 1026 & 1259 & 0,040 & 1058 \\
\hline Porto Alegre & 1595 & 1855 & 2115 & 0,023 & 1880 \\
\hline Recife & 1169 & 1422 & 1722 & 0,025 & 1536 \\
\hline Salvador & 1523 & 1876 & 2229 & 0,023 & 1955 \\
\hline
\end{tabular}

A seguir, os resultados produzidos pelo modelo de simulação foram comparados com trabalhos previamente publicados sobre o tema. Os resultados produzidos pelo modelo de simulação, ver Tabela 7, foram então comparados com a capacidade atual de cada um dos aeroportos escolhidos. A capacidade ideal de cada subsistema, produzida pelo modelo de simulação, foi comparada com a capacidade atual dos aeroportos avaliados, gerando um índice que permitiu agrupar os aeroportos em três classes, conforme descrito a seguir:

1. Situação adequada, quando o índice de utilização menor que $80 \%$;

2. Situação preocupante, quando o índice de utilização estiver entre $81 \%$ e $100 \%$; e,

3. Situação crítica, quando o índice de utilização for superior a $101 \%$. 
Um modelo de simulação para gestão da capacidade dos aeroportos brasileiros

Tabela 7 - Validação do modelo

\begin{tabular}{|c|c|c|c|c|c|c|c|c|c|}
\hline \multirow{2}{*}{$\begin{array}{c}\text { Subsistema } x \\
\text { Aeroporto }\end{array}$} & \multicolumn{3}{|c|}{ Pista (movimentos/hora) } & \multicolumn{3}{|c|}{ Terminal de Passageiros $\left(\mathrm{m}^{2}\right)$} & \multicolumn{3}{|c|}{ Pátio ( $\mathrm{n}^{\circ}$ de posições) } \\
\hline & 2012 & Simulado & Situação & 2012 & Simulado & Situação & 2012 & Simulado & Situação \\
\hline Brasília & 44 & 33 & Adequada & 80.000 & 85.897 & Critica & 32 & 48 & Critica \\
\hline Campinas & 28 & 12 & Adequada & 8.720 & 21.000 & Critica & 8 & 14 & Critica \\
\hline Confins & 29 & 16 & Adequada & 53.950 & 44.725 & Preocupante & 25 & 22 & Preocupante \\
\hline Cuiabá & 22 & 13 & Adequada & 5.460 & 20.418 & Critica & 27 & 18 & Adequada \\
\hline Curitiba & 24 & 15 & Adequada & 45.000 & 39.431 & Preocupante & 18 & 17 & Preocupante \\
\hline Fortaleza & 27 & 12 & Adequada & 38.500 & 39.861 & Critica & 14 & 14 & Preocupante \\
\hline Galeão & 40 & 32 & Adequada & 280.681 & 74.733 & Adequada & 53 & 39 & Adequada \\
\hline Guarulhos & 45 & 39 & Preocupante & 175.000 & 139.724 & Adequada & 63 & 53 & Preocupante \\
\hline Manaus & 25 & 17 & Adequada & 39.484 & 34.161 & Preocupante & 15 & 14 & Preocupante \\
\hline Natal & 27 & 11 & Adequada & 18.013 & 23.303 & Critica & 25 & 15 & Adequada \\
\hline Porto Alegre & 24 & 18 & Adequada & 53.140 & 41.414 & Adequada & 25 & 18 & Adequada \\
\hline Recife & 29 & 14 & Adequada & 52.000 & 38.399 & Adequada & 26 & 19 & Adequada \\
\hline Salvador & 26 & 21 & Preocupante & 60.000 & 43.061 & Adequada & 24 & 29 & Critica \\
\hline
\end{tabular}

Fonte: Elaborado pelos autores

Numa primeira análise da Tabela 7, pode-se verificar que o subsistema em melhor situação é a Pista, pois na maioria dos casos a situação é adequada e em dois casos a situação é preocupante. No entanto, considerando que o subsistema Pista deve ter uma utilização máxima de $80 \%$, conforme recomendação da INFRAERO, pode-se verificar que a situação dos aeroportos de Brasília, Guarulhos, Porto Alegre, Salvador e Galeão passa a ser preocupante, conforme já havia sido previsto por Mckinsey \& Company (2010) para os quatros primeiros anteriormente citados e para Campinas.

Em relação aos resultados do subsistema Pátio, pode-se verificar que a situação é pior, quanto comparada a situação do subsistema Pista. Esta situação já estava prevista em Mckinsey \& Company (2010) e Carvalho (2006). Porém há uma pequena diferença em relação aos resultados encontrados, Mckinsey \& Company (2010) consideram a situação do Pátio dos aeroportos de Cuiabá, Guarulhos e Natal como crítica.

Por ultimo, o subsistema que apresentou o pior desempenho foi o Terminal de Passageiros, situação semelhante indicada por Mckinsey \& Company (2010), Medeiros (2004) e Carvalho (2006). Em relação à Mckinsey \& Company (2010), a principal diferença encontra-se na situação dos aeroportos de Confins, Guarulhos, Porto Alegre e Salvado, onde o a situação destes aeroportos é classificada como crítica. 
Por fim, pode-se dizer que uma pequena diferença de resultados em relação a Mckinsey \& Company (2010) era esperado, dado que em relação ao dimensionamento do terminal de passageiros, Mckinsey \& Company (2010) utilizam a demanda anual de passageiros e não a hora-pico, como no presente trabalho. Além disso, os autores não definem as capacidades de cada subsistema, nem os valores de capacidade calculados. Por fim, deve-se ressaltar que todos os dados utilizados na etapa de validação do modelo são reais e foram cuidadosamente validados em relação aos dados reais já publicados por agências especializadas do setor.

\section{ANÁLISE DE CENÁRIOS}

A análise de cenários foi a última etapa conduzida durante a realização deste trabalho. No modelo proposto, todas as variáveis de dimensionamento estão relacionadas com a Demanda Anual de Passageiros. Desse modo, os dois cenários propostos e analisados nesta seção apresentam variações para esta variável. Cada subsistema foi classificação como adequado, preocupante ou crítico, conforme descrito na seção 3.4. A capacidade prevista de cada subsistema, foi estimada a partir dos investimentos descritos na Tabela 2.

O primeiro cenário simulado neste trabalho considera a avaliação dos analistas do setor aéreo para a demanda de passageiros nos aeroportos brasileiros, onde há a projeção de uma taxa de crescimento da demanda de passageiros no Brasil de 5\% ao ano, como destacam McKinsey \& Company (2010), Carvalho (2006) e ANAC (2007), por exemplo. Os resultados da simulação para o período de 2014 a 2020 são apresentados na Tabela 8.

Analisando a situação desses aeroportos, pode-se verificar que, na hora-pico, todos os aeroportos analisados precisam ampliar a capacidade de pelo menos um dos seus subsistemas, seja por estar em situação preocupante, seja por estar em situação crítica. O Terminal de Passageiros e o Pátio, pela ordem, são os subsistemas em situação mais crítica, enquanto que o subsistema Pista apresentou melhor dimensionado para atender a demanda na hora-pico, mesmo assim, cinco aeroportos finalizaram o período de simulação em situação crítica.

Conforme mencionado, o subsistema Terminal de Passageiros apresentou-se como o mais crítico nos aeroportos analisados, apresentando doze (12) aeroportos em situação crítica e apenas um em situação adequada. A seguir, de acordo com as simulações realizadas para o cenário mais provável, o segundo subsistema mais crítico é o Pátio, pois apresentou oito (8) aeroportos em situação crítica, três (3) em situação preocupante e dois (2) em situação adequada. Quando se compara a situação da Pista e do Pátio dos aeroportos estudados, 
Um modelo de simulação para gestão da capacidade dos aeroportos brasileiros

verifica-se que a situação crítica é comum em quatro (4) deles (Brasília, Guarulhos, Porto Alegre e Salvador) e que a situação adequada é comum em dois (2) deles (Campinhas e Fortaleza). Por último, a Pista é o subsistema que apresenta-se em melhor situação, quando comparado ao demais subsistemas, pois cinco (5) aeroportos encontram-se em situação adequada, três (3) aeroportos encontram-se em situação preocupante e cinco (5) encontram-se em situação crítica na hora-pico.

Tabela 8 - Resultado da simulação para cenário futuro (até 2020)

\begin{tabular}{|c|c|c|c|c|c|c|c|c|c|}
\hline \multirow{2}{*}{$\begin{array}{c}\text { Subsistema } x \\
\text { Aeroporto }\end{array}$} & \multicolumn{3}{|c|}{ Pista (movimentos/hora) } & \multicolumn{3}{|c|}{ Terminal de Passageiros $\left(\mathrm{m}^{2}\right)$} & \multicolumn{3}{|c|}{ Pátio (nº de posições) } \\
\hline & Previsto & Simulado & Situação & Previsto & Simulado & Situação & Previsto & Simulado & Situação \\
\hline Brasília & 44 & 46 & Critica & 80.000 & 173.619 & Critica & 40 & 68 & Critica \\
\hline Campinas & 28 & 17 & Adequada & 30.000 & 31.947 & Critica & 41 & 23 & Adequada \\
\hline Confins & 29 & 22 & Adequada & 53.950 & 65.947 & Critica & 25 & 31 & Critica \\
\hline Cuiabá & 22 & 19 & Preocupante & 5.460 & 30.607 & Critica & 11 & 28 & Critica \\
\hline Curitiba & 24 & 21 & Preocupante & 45.000 & 57.998 & Critica & 10 & 30 & Critica \\
\hline Fortaleza & 27 & 16 & Adequada & 35.660 & 55.085 & Critica & 39 & 24 & Adequada \\
\hline Galeão & 40 & 44 & Critica & 280.681 & 126.203 & Adequada & 62 & 60 & Preocupante \\
\hline Guarulhos & 45 & 50 & Critica & 175.000 & 213.979 & Critica & 61 & 72 & Critica \\
\hline Manaus & 25 & 24 & Preocupante & 39.484 & 57.476 & Critica & 34 & 30 & Preocupante \\
\hline Natal & 27 & 16 & Adequada & 18.013 & 39.321 & Critica & 26 & 23 & Preocupante \\
\hline Porto Alegre & 24 & 26 & Critica & 53.100 & 70.197 & Critica & 25 & 36 & Critica \\
\hline Recife & 29 & 19 & Adequada & 52.000 & 56.776 & Critica & 21 & 26 & Critica \\
\hline Salvador & 25 & 33 & Critica & 69.750 & 72.470 & Critica & 24 & 44 & Critica \\
\hline
\end{tabular}

Com isso, diante dos resultados obtidos, pode-se dizer que os investimentos previstos e/ou realizados para os aeroportos brasileiros, como apontado pela INFRAERO (2013) e TCU (2012), são ainda insuficientes para atender uma demanda com crescimento de $5 \%$ ao ano. Além disso, pode-se verificar que os subsistemas dos aeroportos brasileiros não estão balanceados para atender a demanda na hora-pico, conforme demonstrou-se com as simulações apresentadas ao longo desta seção e também pode-se verificar em relatórios técnicos com ampla circulação nacional, tais como ANAC (2007) e Mckinsey \& Company (2010).

A seguir, analisou-se a situação dos três maiores aeroportos que fazem parte da amostra deste trabalho para verificar a evolução da utilização dos seus subsistemas até o ano de 2030. Os resultados obtidos estão apresentados na Tabela 9, onde os dados são apresentados em notação percentual, o sistema está saturado quando a utilização for superior a 
100\%. A idéia deste experimento foi verificar até que ponto a capacidade atual destes aeroportos consegue suportar o crescimento anual de 5\% da demanda anual de passageiros. De acordo com os dados da Tabela 9, o Pátio e o Terminal de Passageiros do aeroporto de Brasília já estão com sua capacidade esgotada em 2013, dado que sua utilização é superior a $100 \%$, enquanto que a Pista tem capacidade suficiente para atender a demanda até o ano de 2026. O aeroporto do Galeão apresenta-se em situação mais confortável, pois a Pista tem capacidade suficiente para atender a demanda até 2025, o Pátio até 2026 e o Terminal de Passageiros tem capacidade suficiente para atender a demanda até 2030 na demanda hora-pico projetada. Por fim, o aeroporto de Guarulhos apresenta a Pista como o subsistema em melhor situação, tendo capacidade suficiente para atender a demanda projetada até 2023, seguido do Terminal de Passageiros com capacidade suficiente até 2018 e, por último, o Terminal de Passageiros com capacidade suficiente até 2016.

Tabela 9 - Projeção da utilização dos subsistemas dos aeroportos estudados

\begin{tabular}{c|c|c|c|c|c|c|c|c|c}
\hline \multirow{2}{*}{ Ano } & \multicolumn{3}{|c|}{ Brasília } & \multicolumn{3}{|c|}{ Galeão } & \multicolumn{3}{c}{ Guarulhos } \\
\cline { 2 - 10 } & Pista & Pátio & TPS & Pista & Pátio & TPS & Pista & Pátio & TPS \\
\hline 2013 & $73,0 \%$ & $108,0 \%$ & $149,0 \%$ & $73,0 \%$ & $61,0 \%$ & $28,0 \%$ & $77,0 \%$ & $70,0 \%$ & $84,0 \%$ \\
\hline 2014 & $74,0 \%$ & $125,0 \%$ & $157,0 \%$ & $75,0 \%$ & $87,0 \%$ & $30,0 \%$ & $79,0 \%$ & $80,0 \%$ & $89,0 \%$ \\
\hline 2015 & $76,0 \%$ & $138,0 \%$ & $167,0 \%$ & $77,0 \%$ & $73,0 \%$ & $32,0 \%$ & $81,0 \%$ & $87,0 \%$ & $94,0 \%$ \\
\hline 2016 & $78,0 \%$ & $139,0 \%$ & $163,0 \%$ & $79,0 \%$ & $74,0 \%$ & $31,0 \%$ & $83,0 \%$ & $114,0 \%$ & $92,0 \%$ \\
\hline 2017 & $81,0 \%$ & $119,0 \%$ & $171,0 \%$ & $81,0 \%$ & $76,0 \%$ & $33,0 \%$ & $86,0 \%$ & $99,0 \%$ & $97,0 \%$ \\
\hline 2018 & $83,0 \%$ & $132,0 \%$ & $184,0 \%$ & $84,0 \%$ & $66,0 \%$ & $35,0 \%$ & $88,0 \%$ & $95,0 \%$ & $104,0 \%$ \\
\hline 2019 & $85,0 \%$ & $134,0 \%$ & $177,0 \%$ & $86,0 \%$ & $85,0 \%$ & $34,0 \%$ & $90,0 \%$ & $111,0 \%$ & $100,0 \%$ \\
\hline 2020 & $87,0 \%$ & $145,0 \%$ & $187,0 \%$ & $88,0 \%$ & $83,0 \%$ & $36,0 \%$ & $93,0 \%$ & $93,0 \%$ & $106,0 \%$ \\
\hline 2021 & $89,0 \%$ & $132,0 \%$ & $203,0 \%$ & $90,0 \%$ & $78,0 \%$ & $39,0 \%$ & $95,0 \%$ & $102,0 \%$ & $114,0 \%$ \\
\hline 2022 & $92,0 \%$ & $135,0 \%$ & $193,0 \%$ & $93,0 \%$ & $97,0 \%$ & $37,0 \%$ & $97,0 \%$ & $123,0 \%$ & $109,0 \%$ \\
\hline 2023 & $94,0 \%$ & $166,0 \%$ & $206,0 \%$ & $95,0 \%$ & $81,0 \%$ & $39,0 \%$ & $100,0 \%$ & $106,0 \%$ & $116,0 \%$ \\
\hline 2024 & $96,0 \%$ & $156,0 \%$ & $201,0 \%$ & $98,0 \%$ & $93,0 \%$ & $38,0 \%$ & $103,0 \%$ & $120,0 \%$ & $113,0 \%$ \\
\hline 2025 & $99,0 \%$ & $153,0 \%$ & $211,0 \%$ & $100,0 \%$ & $91,0 \%$ & $40,0 \%$ & $105,0 \%$ & $122,0 \%$ & $119,0 \%$ \\
\hline 2026 & $102,0 \%$ & $175,0 \%$ & $226,0 \%$ & $103,0 \%$ & $103,0 \%$ & $43,0 \%$ & $108,0 \%$ & $110,0 \%$ & $127,0 \%$ \\
\hline 2027 & $104,0 \%$ & $165,0 \%$ & $218,0 \%$ & $105,0 \%$ & $110,0 \%$ & $42,0 \%$ & $111,0 \%$ & $135,0 \%$ & $123,0 \%$ \\
\hline 2028 & $105,0 \%$ & $167,0 \%$ & $217,0 \%$ & $108,0 \%$ & $98,0 \%$ & $44,0 \%$ & $111,0 \%$ & $120,0 \%$ & $122,0 \%$ \\
\hline 2029 & $105,0 \%$ & $165,0 \%$ & $217,0 \%$ & $111,0 \%$ & $100,0 \%$ & $48,0 \%$ & $111,0 \%$ & $132,0 \%$ & $122,0 \%$ \\
\hline 2030 & $105,0 \%$ & $172,0 \%$ & $217,0 \%$ & $111,0 \%$ & $110,0 \%$ & $45,0 \%$ & $111,0 \%$ & $138,0 \%$ & $122,0 \%$ \\
\hline
\end{tabular}

Por fim, repetiram-se os experimentos com um aumento anual de $11 \%$ para a demanda de passageiros. Este valor corresponde ao crescimento médio da movimentação de passageiros nos aeroportos brasileiros nos últimos dez anos. No entanto, para este cenário, o REAd | Porto Alegre - Edição 80 - N 1 - janeiro/abril 2015 - p. 1-26 
Um modelo de simulação para gestão da capacidade dos aeroportos brasileiros

único subsistema que apresentou a situação adequada foi o Terminal de Passageiros do aeroporto de Fortaleza, com utilização de $65 \%$ da capacidade prevista, os demais subsistemas dos aeroportos estudados apresentaram no final das simulações situação preocupante ou crítica.

\section{CONCLUSÕES E CONSIDERAÇÕES FINAIS}

O setor aéreo é importante para o crescimento e desenvolvimento econômico do país. Dessa maneira, a gestão dos aeroportos torna-se um elemento estratégico, sendo necessário oferecer um serviço de qualidade para os passageiros. No decorrer deste trabalho, apresentouse a estruturação de um modelo em Dinâmica de Sistemas para avaliar a capacidade dos aeroportos brasileiros na hora-pico.

O modelo proposto neste trabalho consiste em um método formal para avaliar a capacidade dos subsistemas desses aeroportos e, assim, auxiliar a gestão aeroportuária. A construção de um modelo dessa natureza apresenta limitações, em sua maioria, provocada pela incapacidade de se estabelecer todas as relações existentes entre as variáveis de um sistema, pelo período de tempo analisado, pelas simplificações inerentes a qualquer processo de modelagem e, neste caso em particular, pelo método utilizado para calcular a demanda na hora-pico. Desse modo, deve-se ponderar, ao decidir sobre as melhores ações a serem realizadas na alteração da capacidade dos subsistemas de cada aeroporto, que o funcionamento do modelo considera a demanda na hora-pico, devendo-se tomar cuidado com o superdimensionamento das variáveis envolvidas.

Para efeito de comparação e validação do modelo proposto, os resultados apresentados neste trabalho são semelhantes aos apresentados em trabalhos prévios sobre o tema, tais como Carvalho (2006) e Mckinsey \& Company (2010). Desse modo, a partir da validação do modelo, dos experimentos conduzidos e das análises comparativas realizadas com os dados produzidos, pode-se dizer que o modelo apresentado neste trabalho pode ser utilizado como uma ferramenta de apoio no planejamento do sistema aeroportuário proporcionando adequações na capacidade dos subsistemas dos aeroportos em condições limite.

Entre os trabalhos futuros projetados estão o desenvolvimento de um modelo para previsão de demanda por passagens aéreas, o aprimoramento do modelo de Wang e Pitfield (1999) para cálculo da demanda hora-pico, a modelagem do detalhamento de cada um dos subsistemas analisados neste trabalho, além do estudo de outras dimensões relacionadas à capacidade aeroportuária.

REAd | Porto Alegre - Edição 80 - N 1 - janeiro/abril 2015 - p. 1-26 


\section{AGRADECIMENTOS}

Os autores agradecem aos dois referees anônimos pelas sugestões de melhoria do trabalho, ao CNPq pelo auxilio financeiro para realização desta pesquisa, processo número 472897/20136, e a CAPES pela bolsa de estudos concedida.

\section{REFERÊNCIAS}

ALMEIDA, M. R.; MARIANO, E. B.; REBELATTO, D. A. N. Análise de eficiência dos aeroportos internacionais brasileiros. Revista Produção Online, edição especial, 2007.

ANAC. Estudo de Demanda Detalhada dos Aeroportos Brasileiros. Rio de Janeiro, vol. 1, 2007.

ASHFORD, N.; MUMAYIZ, S.; WRIGHT, P. H. Airport Engineering: Planning, Design, and Development of 21st Century Airports. John Willey \& Sons. New York, $4^{\mathrm{a}}$ ed, 2011.

ASHFORD, N.; STANTON, H. P. M.; MOORE, C. A. Airport operations. John Willey, 1984.

ASSAF, A. G.; GILLEN, D., BARROS, C. Performance assessment of UK airports: Evidence from a Bayesian dynamic frontier model. Transportation Research Part E, v. 48, p. 603$615,2012$.

BARROS, C. P.; DIEKE, P. U.C. Measuring the economic efficiency of airports: A SimarWilson methodology analysis. Transportation Research Part E, v. 44, p. 1039-1051, 2008.

CARVALHO, B. G. Uma metodologia para obtenção de um diagnóstico dos principais aeroportos no Brasil através da avaliação da demanda e capacidade. Tese de Doutorado. São José dos Campos: ITA, 2006.

CORREIA, A. R.; WIRASINGHE, S. C.; BARROS, A. G. Overall level of service measures for airport passenger terminals. Transportation Research Part A, v. 42, p. 330-346, 2008.

CURI, C.; GITTO, S.; MANCUSO, P. The Italian airport industry in transition: a performance analysis. Journal of Air Transport Management, v. 16, p. 218-221, 2010.

FERNANDES, E.; PIRES, H. M. Malmquist financial efficiency analysis for airlines. Transportation Research. Part E, Logistics and Transportation Review, v. 48, p. 1049-1055, 2012.

FERNANDES, E.; PACHECO, R. R. A quality approach to airport management. Quality \& Quantity, v. 44, p. 551-564, 2010. 
Um modelo de simulação para gestão da capacidade dos aeroportos brasileiros

FORRESTER, J. W. Industrial Dynamics. Cambridge, MIT Press, 1961.

GILBO, E. P. Airport Capacity: Representation, Estimation, Optimization. IEEE Transactions on Control Systems Technology, v. 1, n. 3, 1993.

GILLEN, D.; LALL, A. Developing measures of airport productivity and performance: An application of Data Envelopment Analysis. Transportation Research Part E, v. 33, n. 4, p. 261-273, 1997.

HOOPER, P. G.; HENSHER, D. A. Measuring total factor productivity of airports - an index number approach.Transportation Research Part E, v. 33, n. 4, p. 249-259, 1997.

HORONJEFF, R.; MCKELVEY, F. X.; SPROUlE, W. J.; YOUNG, S. B. Planning and Design of Airports. McGraw Hill, $5^{\mathrm{a}}$ Ediç̧ão, 2010.

INFRAERO. Disponível em: <http://www.infraero.gov.br/> Acesso em: 02. abril. 2013.

KIYILDI, R. K.; KARASAHIN, M.The capacity analysis of the check-in unit of Antalya Airport using the fuzzy logic method.Transportation Research Part A, v. 42, p. 610-619, 2008.

KUO, M. S.; LIANG, G. S. Combining VIKOR with GRA techniques to evaluate service quality of airports under fuzzy environment. Expert Systems with Applications, v. 38, p. 1304-1312, 2011.

MANATAKI I. E., ZOGRAFOS, K. G. A generic system dynamics based tool for airport terminal performance analysis. Transportation Research Part C, v. 17, p. 428-443, 2009.

MANATAKI I. E., ZOGRAFOS, K. G. Assessing airport terminal performance using a system dynamics model. Journal of Air Transport Management, v. 16, p. 86-93, 2010.

MARAZZO, M.; SCHERRE, R.; FERNANDES, E. Air transport demand and economic growth in Brazil: A time series analysis. Transportation Research Part E, v. 46, p. 261-269, 2010 .

MARTÍN, J. C.; ROMÁN, C. A benchmarking analysis of Spanish commercial Airports: A comparison between SMOP and DEA ranking methods. Networks \& Spatial Economics, v. 6, p. 111-134, 2006.

MARTÍN, J. C.; ROMÁN, C.An application of DEA to measure the efficiency of Spanish airports prior to privatization. Journal of Air Transport Management, v. 7, p. 149-157, 2001.

MCKINSEY \& COMPANY. Estudo do Setor de Transporte Aéreo do Brasil. Banco Nacional de Desenvolvimento Econômico e Social, 2010.

REAd | Porto Alegre - Edição 80 - N 1 - janeiro/abril 2015 - p. 1-26 
MEDEIROS, A. G. M. Um método para dimensionamento de terminais de passageiros em aeroportos brasileiros. Dissertação de Mestrado. São José dos Campos: Instituto Tecnológico de Aeronáutica, 2004.

MELLO, J. C. C. B. S.; MEZA, L. A.; GOMES, E. G.; BIONI NETO, L. Curso de Análise de Envoltória de Dados. In: Anais XXXVII Simpósio Brasileiro de Pesquisa Operacional (SBPO), 2005.

MILLER, B.; CLARKE, J. P. The hidden value of air transportation infrastructure. Technological Forecasting \& Social Change, v. 74, p. 18-35, 2007.

OUM, T. H.; YU, C.; FU, X. A comparative analysis of productivity performance of the world's major airports: summary report of the ATRS global airport benchmarking research report - 2002. Journal of Air Transport Management, v. 9, p. 285-297, 2003.

PACHECO, R. R.; FERNANDES, E.; SANTOS, M. P. S. Management style and airport performance in Brazil. Journal of Air Transport Management, v. 12, p. 324-330, 2006.

REYNOLDS-FEIGHAN, A. J.; BUTTON, K. J. An assessment of the capacity and congestion levels at European Airports. Journal of Air Transport Management, v. 5, p. 113-134, 1999.

SARKIS, J.; TALLURI, S. Performance based clustering for benchmarking of US airports. Transportation Research Part A, v. 38, p. 329-346, 2004

SALGADO, L. H.; VASSALLO, M.; OLIVEIRA, A. Regulação, Politicas Setoriais, Competitividade e Formação de Preços: Considerações sobre o Transporte Aéreo no Brasil. Revista de Literatura dos Transportes, v. 4, p. 7-48, 2010.

STERMAN, J. D. Business Dynamics: System Thinking and Modeling for a Complex World. USA: McGraw-Hill Higher Education, 2000.

SURYANI, E., CHOU, S. Y.; CHEN, C. H. Air passenger demand forecasting and passenger terminal capacity expansion: A system dynamics framework. Expert Systems with Applications, v. 37, p. 2324-2339, 2010.

SURYANI, E., CHOU, S. Y.; CHEN, C. H. Dynamic simulation model of air cargo demand forecast and terminal capacity planning. Simulation Modelling Practice and Theory, v. 28, p. 27-41, 2012. 
Um modelo de simulação para gestão da capacidade dos aeroportos brasileiros

TCU - Portal de Fiscalização da Copa 2014. Disponível em: <http://portal2.tcu.gov.br/portal/page/portal/TCU/copa2014/documentos>. Acesso em: 30. novembro. 2012.

WANG, P. T., PITFIELD, D. E. The derivation and analysis of the passenger peak hour: an empirical application to Brazil. Journal of Air Transport Management, v. 5, p.135-141, 1999.

WANKE, P. F. Capacity shortfall and efficiency determinants in Brazilian airports: Evidence from bootstrapped DEA estimates. Socio-Economic Planning Sciences, v. xxx, p. 1-14, 2012.

YEH, C. H.; KUO, Y. L. Evaluating passenger services of Asia-Pacific international airports. Transportation Research Part E, v. 39, p. 35-48, 2003.

YU, M. M. Assessment of airport performance using the SBM-NDEA model. Omega, v. 38, p. 440-452, 2010.

YU, M. M. Assessment of airport performance using the SBM-NDEA model. Omega, v. 38, p. $440-452,2010$.

ZHANG, B.; WANG, J.; LIU, C.; ZHAO, Y. Evaluating the technical efficiency of Chinese airport airside activities. Journal of Air Transport Management, v. 20, p. 23-27, 2012. 